\title{
Gas Separation Membrane Material Selection Criteria: Weakly and Strongly Interacting Feed Component Situations
}

\author{
W. J. Koros and D. R. B. WALKER \\ Department of Chemical Engineering, The University of Texas at Austin, \\ Austin, Texas, USA 78712
}

(Received November 26, 1990)

\begin{abstract}
Materials from the polyimide and polycarbonate families with an attractive combination of productivity and permselectivity for important gas pairs have been reported recently. A systematic group of materials from both of these families will be considered in this paper. Material optimization strategies in the absence of strong interactions between the membrane material and the penetrating gas mixture will also be discussed in the context of these materials. In addition, a material from a relatively unexplored family of rigid chain polymers, the polypyrrolones, will be compared to the behavior of the polycarbonates and polyimides. Performance for mixtures such as carbon dioxide and methane that interact relatively strongly will be contrasted those such as oxygen and nitrogen that interact weakly with the membrane materials. KEY WORDS Polycarbonates / Polyimides / Polypyrrolone / Permeation / Gas Separation / Sorption / Diffusion
\end{abstract}

The permeability, $\mathbf{P}$, of a penetrant through the selective layer of a gas separation membrane can be expressed as the product of an effective solubility of the penetrant in the polymer matrix, $\mathbf{S}$, and an average diffusivity of the penetrant through the polymer matrix, D 1 .

$$
\mathbf{P}=\mathbf{D} \mathbf{S}
$$

For conditions of negligible downstream pressure such as those considered here, the solubility coefficient, $\mathbf{S}$, equals the secant slope of the gas sorption isotherm, $\mathbf{S}=\mathrm{C} / \mathrm{p}$, evaluated at the upstream conditions, and $\mathbf{D}$ is determined as the ratio of $\mathbf{P}$ and $\mathbf{S}$. The solubility coefficient is known to be affected by the inherent condensibility of the penetrant, by polymer-penetrant interactions, and the amount of excess or free volume existing in the glassy polymer ${ }^{1}$.

In addition to permeability, the effects on membrane permselectivity must be considered when structural modifications are proposed to optimize performance. When the downstream pressure is negligible, the separation factor for a mixture of gases $A$ and $\mathrm{B}, \alpha_{\mathrm{A} / \mathrm{B}}$, is equal to the ideal separation factor based on the individual permeabilities of the gases $\mathrm{A}$ and $\mathrm{B}, \alpha_{\mathrm{A} / \mathrm{B}}^{*}$ given by Eq. (3):

$$
\begin{aligned}
& \alpha_{\mathrm{A} / \mathrm{B}}=\frac{\left[\mathrm{y}_{\mathrm{A}} / \mathrm{y}_{\mathrm{B}}\right]}{\left[\mathrm{x}_{\mathrm{A}} / \mathrm{x}_{\mathrm{B}}\right]} \\
& \alpha_{\mathrm{A} / \mathrm{B}}^{*}=\frac{\mathbf{P}_{\mathrm{A}}}{\mathbf{P}_{\mathrm{B}}}
\end{aligned}
$$

where $x_{i}$ 's and $y_{i}$ 's are the mole fractions of components $A$ and $B$ at the upstream and 
downstream sides of the membrane. The ideal separation factor, $\alpha_{\mathrm{A} / \mathrm{B}}^{*}$ provides a measure of the intrinsic permselectivity of a membrane material for mixtures of $\mathrm{A}$ and $\mathrm{B}$. In the absence of plasticizing effects due to strong polymer-penetrant interactions, $\alpha_{\mathrm{A} / \mathrm{B}}$ in mixed gas situations generally is approximated using the ratio of permeabilities of pure gases $\mathrm{A}$ and $\mathrm{B}^{2}$. If $\mathrm{Eq}(1)$ is substituted into $\mathrm{Eq}$ (3), the ideal separation factor can be factored into diffusivity selectivity, and solubility sélectivity, factors, viz. :

$$
\alpha_{\mathrm{A} / \mathrm{B}}^{*}=\left[\frac{\mathbf{D}_{\mathrm{A}}}{\mathbf{D}_{\mathrm{B}}}\right]\left[\frac{\mathbf{S}_{\mathrm{A}}}{\mathbf{S}_{\mathrm{B}}}\right]
$$

The diffusivity selectivity reflects the ability of the polymer to discriminate between penetrants on the basis of their sizes and shapes and is governed by intrasegmental motions and intersegmental packing. The solubility selectively is difficult to adjust without causing significant losses in the diffusivity selectivity ${ }^{3}$, so it is necessary to increase the diffusivity selectivity without incurring large reductions in the diffusivity of the desired component, A.

It has been demonstrated that significant increases in diffusivity and diffusivity selectivity can be obtained by simultaneously inhibiting intrasegmental motions and intersegmental chain packing ${ }^{4-6}$. The wide angle $x$-ray diffraction d-spacing, a measure of center-to-center chain distances, characterizes the "openness" of a polymer matrix; however, the ratio of specific free volume to polymer specific volume, the fractional free volume, is often preferred for this purpose. This latter index takes into account the filling of space by bulky side groups, but requires an estimate of the occupied volume of the polymer based on a group contribution method such as that of Bondi or Van Krevelen ${ }^{7-8}$.

\section{MATERIALS}

The polymers discussed in study are shown in Table I. The synthesis and characterization of all of the materials have been discussed separately in some detail in previous papers ${ }^{4-6}$. The objective here is to summarize and compare properties between the various materials in the present discussion.

\section{DISCUSSION}

\section{Identification of improved membrane materials.}

Permeabilities vs. selectivities of various gas pairs are plotted in Fig. 1 as a solid line to reflect attainable properties when using commercial polymers that might be considered for gas separation applications without resorting to molecular design considerations such as those being discussed here ${ }^{4-6}$. The typical tradeoff between high productivity and lower selectivity is clearly seen in this plot. Two families, the polycarbonates and polyimides, have been studied intensively and are shown on this plot as symbols for comparison to the line defined by the commercial polymers. In addition, a third material somewhat related to the polyimides, a polypyrrolone is also shown in this figure. The various structures are further identified in Table I by symbols and their "short hand" names to allow their identification in Fig. 1 and for use in later discussions in the text. Clearly several of the tailored materials lie in the desirable region above the line representing commercial materials.

\section{Polycarbonates.}

To allow more detailed consideration, the permeabilities and selectivities of the systematically varied polycarbonates are reported in Tables II for the $\mathrm{CO}_{2} / \mathrm{CH}_{4}$ and the $\mathrm{O}_{2} / \mathrm{N}_{2}$ systems, respectively. The solubility and diffusivity contributions to the 
permeability and selectivity can be discussed in terms of parameters in Table III. For instance, the degree of "openness" of the matrix can be characterized by (i) average interchain spacing between the backbones of the amorphous matrix (d-spacings) and,

(ii) fractional free volume fraction (FFV) as well as chain rigidity. The intrinsic resistance to segmental motion can be characterized by (ii) the onset temperature (Tg) for large scale rotational motions at the specified $1 \mathrm{~Hz}$ testing rate, and (iii) the onset temperature for sub$\mathrm{Tg}$ transition $(\mathrm{T} \gamma)$ temperatures at $1 \mathrm{~Hz}$. This later temperature is an indicator of the

Table I: Polymers discussed in this study.

\begin{tabular}{|c|c|}
\hline $\begin{array}{l}\text { Standard } \\
\text { bisphenol-A } \\
\text { Polycarbonate } \\
\text { PC }\end{array}$ & $0-\left.\right|_{\mathrm{CH}_{3}} ^{\mathrm{CH}_{3}} \bigcirc$ \\
\hline $\begin{array}{l}\text { Hexafluorinated } \\
\text { bisphenol-A } \\
\text { Polycarbonate } \\
\text { HFPC }\end{array}$ & 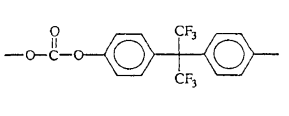 \\
\hline $\begin{array}{l}\text { Tetramethyl } \\
\text { bisphenol-A } \\
\text { Polycarbonate } \\
\text { TMPC }\end{array}$ & \\
\hline $\begin{array}{l}\text { Hexafluorinated } \\
\text { Tetramethyl } \\
\text { bisphenol-A } \\
\text { Polycarbonate } \\
\text { TMHFPC }\end{array}$ & $=\gamma_{\mathrm{CH}_{3}}^{\mathrm{CH}_{3}}$ \\
\hline
\end{tabular}

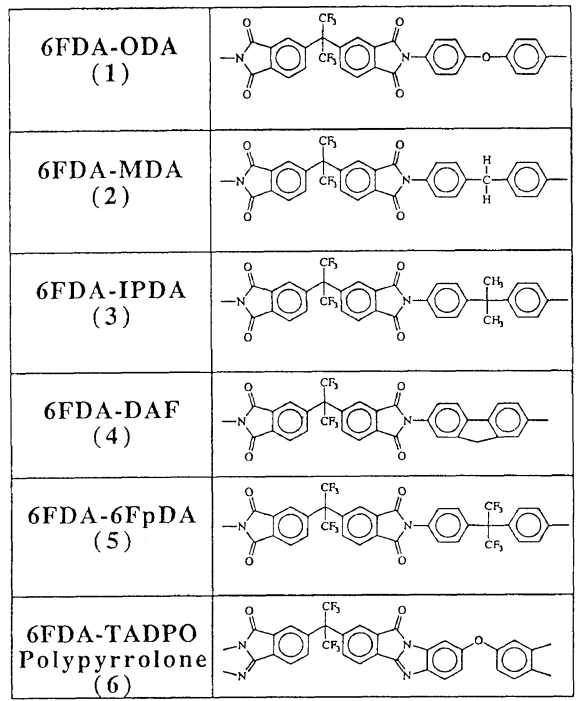

Figure 1: Tradeoff between permselectivity and permeability at $35^{\circ} \mathrm{Cfor}$ the separation of two important gas pairs, $\mathrm{CO}_{2} / \mathrm{CH}_{4}$ and $\mathrm{O}_{2} / \mathrm{N}_{2}$. The symbols refer to materials in Table $\mathrm{I}$.
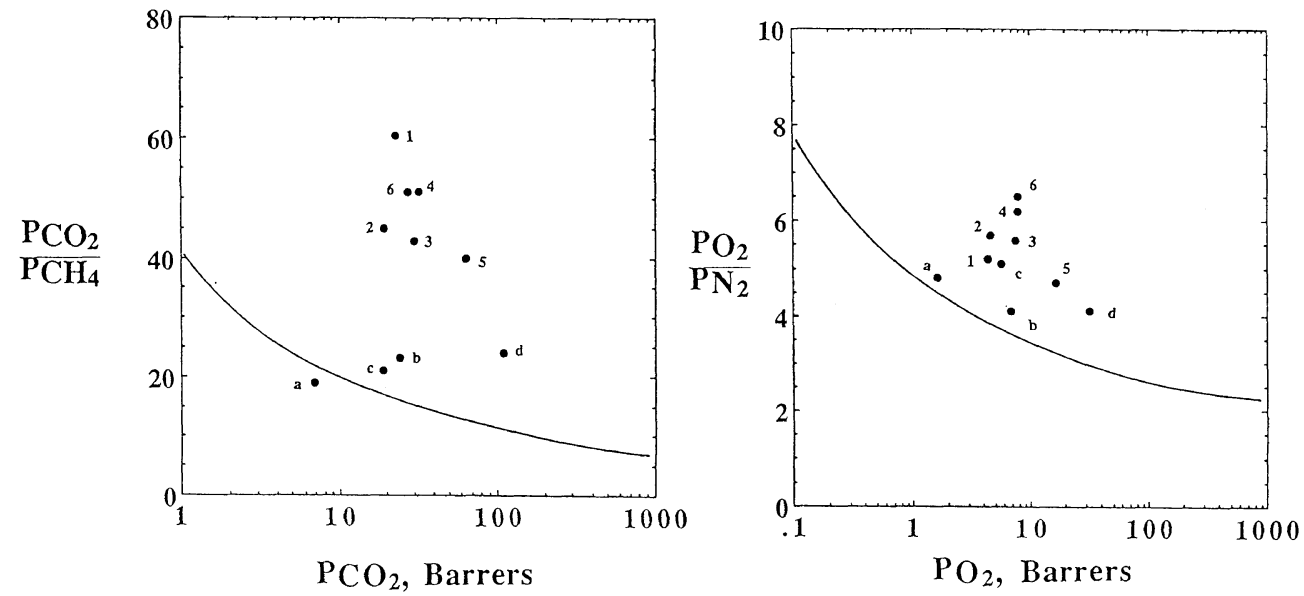
ability of the repeat unit to undergo torsional rocking motions around the carbonate hinge points. The presence of these motions are expected to promote diffusion of small molecules for this systematically varied series of materials.

The largest d-spacing occurs in the rigid packing inhibited TMHFPC, followed by TMPC, HFPC and finally the standard PC. Relative differences between polymer segmental mobility within the group also bears consideration. The effects on the permeability and permselectivity caused by differences in segmental mobility of the different materials may be especially significant for two materials with similar d-spacings. Both standard PC and HFPC have a $\mathrm{T} \gamma$ of $-100^{\circ} \mathrm{C}$ while both TMPC and TMHFPC have a $\mathrm{T} \gamma$ of $+50^{\circ} \mathrm{C}$, indicating that the hexafluoro substitution does not strongly hinder torsional motion about the carbonate linkages while the tetramethyl substitutions surrounding the carbonate linkage greatly inhibit its ability to undergo torsional motions, as is reflected by the $150^{\circ} \mathrm{C}$ higher value of $\mathrm{T} \gamma$ for both TMPC and TMHFPC compared. These data are consistent with space filling models of the various structures as well.

The large d-spacing difference between TMPC and the unsubstituted PC gives this material a larger diffusion coefficient for $\mathrm{CO}_{2}$ than in PC despite its significantly inhibited motions which provide TMPC's higher diffusivity selectivity compared to both PC and HFPC. Equally interesting is the fact that TMHFPC has essentially an equivalent mobility selectivity to that for TMPC for $\mathrm{CO}_{2} / \mathrm{CH}_{2}$ although it has both a much higher diffusivity and permeability.

Unlike the $\mathrm{CO}_{2} / \mathrm{CH}_{4}$ pair, $\mathrm{O}_{2}$ and $\mathrm{N}_{2}$ have very similar molecular dimensions, differing by less than $0.2 \AA 1$, thereby placing very stringent demands upon the mobility selecting ability of the polymer. For this latter pair, when the additional opening of the
Table II: Gas separation properties for polycarbonates in Table I.

\begin{tabular}{|c|c|c|c|c|c|c|}
\hline Polymer & ${ }^{{ }^{(1}}$ & $\begin{array}{l}\mathrm{PCO}_{2} \\
\mathrm{P}^{\mathrm{P} C \mathrm{H}_{4}}\end{array}$ & $\mathrm{SCO}_{2}^{12}$ & $\begin{array}{l}\mathrm{S}^{\mathrm{SCO}_{2}} \\
\mathrm{SCH}_{4}\end{array}$ & $\mathrm{DCO}_{2}^{13}$ & $\begin{array}{l}\mathrm{DCO}_{2} \\
\mathrm{D}^{\mathrm{D}} \mathrm{H}_{4} \\
\end{array}$ \\
\hline$-a-c-a-<$ & 6.8 & 19 & 1.6 & 4.0 & 3.2 & 4.7 \\
\hline & 24 & 23 & 2.7 & 3.9 & 6.7 & 5.9 \\
\hline & 19 & 21 & 2.6 & 3.2 & 5.4 & 6.6 \\
\hline & 110 & 24 & 4.1 & 3.4 & 21 & 7.0 \\
\hline
\end{tabular}

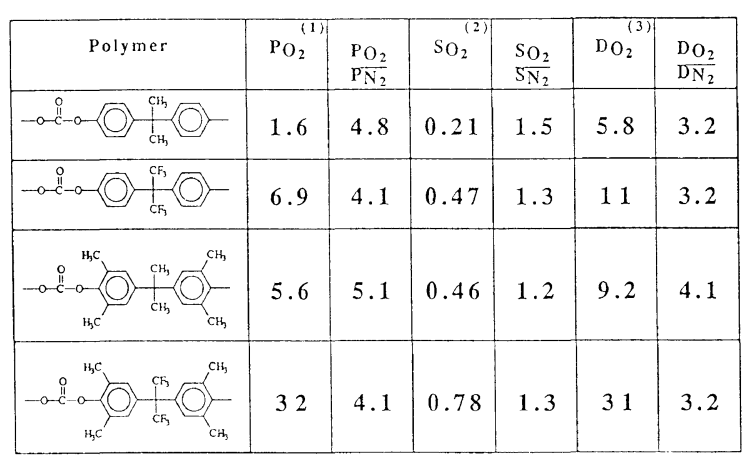

$\begin{array}{llll}\text { (1) Barrer } & \text { (2) } \mathrm{cc}(\mathrm{STP}) / \mathrm{cc} \text { atm } & \text { (3) } 10^{-8} \mathrm{~cm}^{2} / \mathrm{sec}\end{array}$

Table III: Physical properties for polycarbonates in Table I.

\begin{tabular}{|c|c|c|c|c|}
\hline Polymer & $\begin{array}{c}\mathrm{Tg} \\
{ }^{\circ} \mathrm{C}\end{array}$ & $\begin{array}{c}\mathrm{T} \gamma \\
{ }^{\circ} \mathrm{C}\end{array}$ & $\begin{array}{c}\text { d-space } \\
\mathrm{A}\end{array}$ & $\mathrm{FFV}$ \\
\hline 0 & 150 & -100 & 5.2 & 0.164 \\
\hline & 176 & -100 & 5.8 & 0.195 \\
\hline
\end{tabular}


matrix is carried out by combining both the tetramethyl and hexafluoro substitutions, the inhibition of torsional motion in the TMHFPC is insufficient to maintain the same diffusivity selectivity found in TMPC.

Polyimides vs. Polycarbonates. Comparisons across families of materials is somewhat difficult and can be a misleading if done casually; however, within limits, some insights are possible. The permeability and solubility data measured for two polyimides are presented in Table IV for comparison to two polycarbonates. For both gas pairs, the polyimides corresponding to the isopropylidene and fluorinated isopropylidene units

Table IV: Transport property comparison between polycarbonates and related polyimides.

have markedly higher permeabilities for the desired product gases while having higher permselectivities than the polycarbonates.

Detailed comparisons between the corresponding polycarbonate and polyimide materials (e.g., PC vs 6FDA-IPDA, and HFPC vs 6FDA6FpDA) are useful. Clearly, the primary differences in permeabilities between the two classes lies not so much in their diffusivities to the

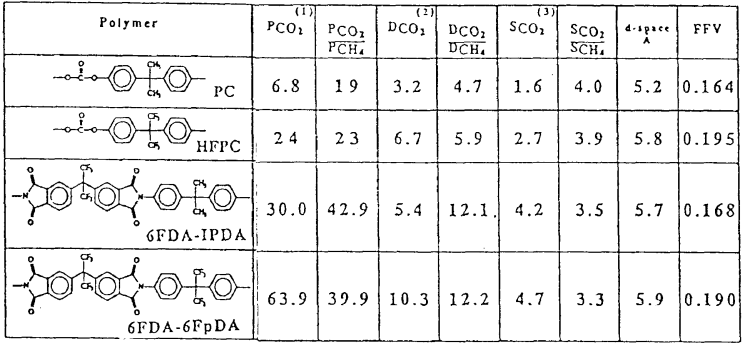

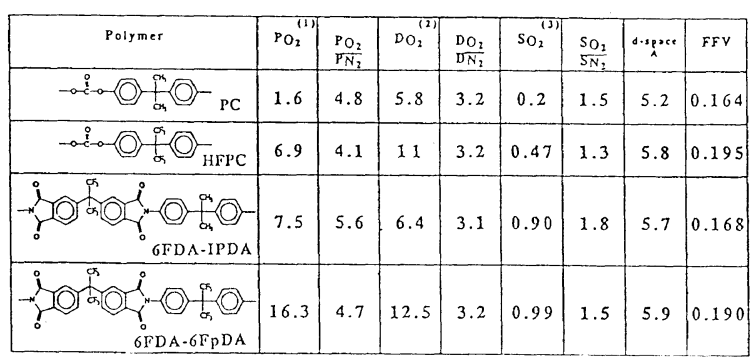

(1) Barrer (2) $10.8 \mathrm{~cm}^{2} / \mathrm{sec}$ (3) $\mathrm{cc}(\mathrm{STP}) / \mathrm{cc}$ atm desired product gases, but in their solubilities which are markedly higher in the polyimides.

While the absolute solubilities are higher in the polyimides, the solubility selectivities are not very different in the polycarbonates and polyimides. For the $\mathrm{CO}_{2} / \mathrm{CH}_{4}$ system, the significantly higher permselectivities of the polyimides arise from their superior mobility selectivity abilities. Similar superiority is not apparent in the case of the $\mathrm{O}_{2} / \mathrm{N}_{2}$ system. Based on a comparison of the d-spacings and fractional free volumes, the similarity in diffusivities and diffusivity selectivity might be expected, so the unusually high mobility selectivity for the $\mathrm{CO}_{2} / \mathrm{CH}_{4}$ system is somewhat surprising. Presumably, this difference between the families reflects the extraordinary sensitivity to subtle motional factors and the distribution of free volume as well as the total fraction of free volume characterized by FFV. We speculate that the higher absolute solubilities for all gases in the polyimides as compared to the polycarbonates with similar free volume fractions and dspacings also may reflect subtle differences in the distribution of free volume in the two families. Rather heterogeneous segmental or even subsegmental scale regions of imperfectly packed and efficiently packed environments are more prone to exist in the rigid polyimides as opposed to the more flexible polycarbonates. In this case, the overall fraction of unoccupied volume and average d-spacing could still be similar in the two types of materials. Nevertheless, if a larger percentage of the overall FFV is distributed as penetrant scale packing defects, the ability to accommodate a penetrant with negligible endothermic requirement to form a "sorbed cage" would promote sorption in the polyimides. 
Consistent with the observed data, such a situation would promote sorption of all penetrants, but the solubility selectivity would be affected little. Moreover, if the overall free volume fraction is equivalent in the polycarbonates and polyimides, but a larger portion of this unoccupied volume is distributed as packing defects, the efficiently packed subsegment regions in the polyimides would tend to have relatively less local free volume than for the polycarbonates. This is consistent with the higher mobility selectivity of the polyimides. In this case, the improved size and shape discrimination would occur as penetrants move through these neighboring regions of lower free volume in local equilibrium exchanges with the less well packed regions during the diffusion process.

Thus a higher average solubility for all gases in the packing disrupted matrix, and good size and shape selection during movement through the matrix can be rationalized.

\section{FDA-ODA Polyimide vs 6FDA-TADPO Polypyrrolone.}

Consistent with the expected $\mathrm{CO}_{2}$ interactions with carbonyl groups, the $\mathrm{CO}_{2}$ solubility coefficient in Table $\mathrm{V}$ is greater in the polyimide than in the polypyrrolone. The polyimide contains nearly twice as many carbonyls per unit volume as the polypyrrolone, yet the $\mathrm{CO}_{2}$ solubility coefficient for the polypyrrolone is only $10 \%$ lower than for the polyimide, presumably reflecting the much higher fractional free volume in the pyrrolone. The influence of the carbonyl concentration is more apparent upon examination of the solubility selectivity for $\mathrm{CO}_{2} / \mathrm{CH}_{4}$, since the effects of fractional free volume are largely cancelled in this relative solubility parameter. The solubility selectivity of the polypyrrolone is considerably less than for the polyimide and for all of the polycarbonates. Despite this fact,

Table V: Transport property comparison between polyimides and polypyrrolone based on 6FDA dianhydride.

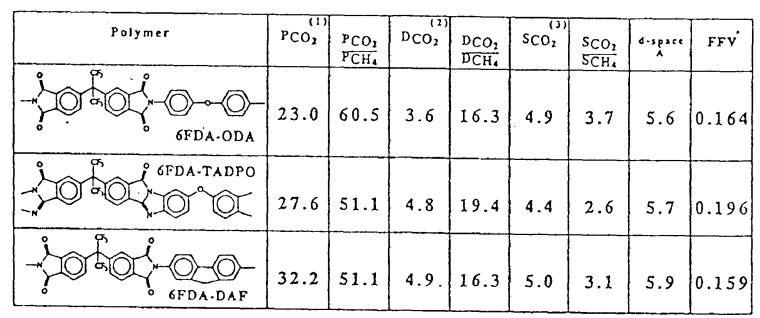
the overall $\mathrm{CO}_{2} / \mathrm{CH}_{4}$ permselectivity of the polypyrrolone is only $15 \%$ lower than for the polyimide and much higher than for the polycarbonates due to the high diffusivity selectivity of the polypyrrolone. The greater diffusivity selectivity of the polypyrrolone relative to the polyimide presumably reflects the suppression of chain segmental motions in the polypyrrolone.

In a fused ring system motions of individual rings require bond distortion. The imide nitrogen

\begin{tabular}{|c|c|c|c|c|c|c|c|c|}
\hline Polymer & $\mathrm{PO}_{2}$ & $\begin{array}{l}\mathrm{PO}_{2} \\
{ }^{\mathrm{P} \mathrm{N}_{2}}\end{array}$ & $\mathrm{DO}_{2}{ }_{2}^{12}$ & 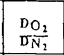 & $\mathrm{SO}_{2}^{(1)}$ & $\frac{S}{S}$ & c.spene & FFV \\
\hline & 4.34 & 5.2 & 3.20 & 2.75 & 1.03 & 1.9 .0 & 5.6 & \\
\hline 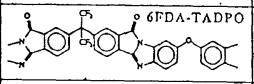 & 7.90 & 6.5 & 5.80 & 5.06 & 1.04 & 1.30 & 5.7 & 0.196 \\
\hline 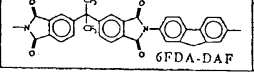 & 7.85 & 6.2 & 4.97 & 3.47 & 1.20 & 1.78 & 5.9 & \\
\hline
\end{tabular}

(1) Barrer
(2) $10^{-8} \mathrm{~cm}^{2} / \mathrm{sec}$ (3) $\mathrm{cc}(\mathrm{STP}) / \mathrm{cc}$ atm atom is only weakly pi-conjugated to the phenyl ring, supporting the possibility of motions involving the phenyl rings of a polyimide based on a para substituted diamine, such as 6FDA-ODA. The polypyrrolones represent a limiting case of chain segment motion suppression due to the inclusion of all phenyl rings as part of fused ring systems.

Interestingly, a large diffusivity selectivity increase of $83 \%$ is seen in going from the polyimide to the pyrrolone for the $\mathrm{O}_{2} / \mathrm{N}_{2}$ pair in Table $\mathrm{V}$. This large increase in the $\mathrm{O}_{2} / \mathrm{N}_{2}$ diffusivity selectivity demonstrates the importance of the suppression of motion in the polypyrrolone, which despite the abundant fractional free volume allows discrimination between penetrants of similar size unlike the situation for the TMHFPC discussed earlier in the polycarbonate family. 
6FDA-DAF Polyimide vs 6FDA-TADPO Polypyrrolone.

The polypyrrolone is slightly less permeable than the DAF polyimide but is slightly more selective. The polyimide has a greater $\mathrm{d}$-spacing than the polypyrrolone, but a smaller fractional free volume as shown in Table V. Although the DAF segment contributes a long, rigid, and flat portion to the polyimide chain, a decrease in packing efficiency results due to the bulky and sterically hindered hexafluoroisopropylidene linkage. The penetrant solubilities and solubility selectivities for the polypyrrolone are lower than for the polyimide, whereas the diffusivities and the diffusivity selectivities of the polypyrrolone are roughly the same as or greater than the polyimide. The similarities in transport properties are not surprising since both polymers contain fused ring systems separated by bulky tetrahedral carbons in the backbone. These structural similarities are what provided the idea for studying the polypyrrolone in the first place?

The backbone of the polypyrrolone contains ether linkages while the 6FDA-DAF contains imide linkages and hexafluoroisopropylidene linkages, but no ether linkages. The motions in the polypyrrolone allowed by the ether linkages most likely would involve nearly one repeat unit, whereas the motions in the polyimide allowed by the imide linkages may involve only a 6FDA segment or a DAF segment.

The solubility selectivity for the $\mathrm{CO}_{2} / \mathrm{CH}_{4}$ gas pair is again greater for the polyimide than for the polypyrrolone, yet the diffusivity selectivity of the polypyrrolone is greater than the polyimide. The two factors counterbalance one another leading to similar permselectivities for the two polymers.

\section{High Pressure $\mathrm{CO}_{2}$ Permeation Results.}

High pressure $\mathrm{CO}_{2}$ permeation for the polypyrrolone, the standard and the hexafluoro polycarbonates as well as the two polyimides discussed above are shown in Fig. 2. The polyimides and the fluorinated polycarbonates show large increases in $\mathrm{CO}_{2}$ permeability at moderate pressures due to facilitation in segmental motions by high sorption levels of $\mathrm{CO}_{2}$. The polypyrrolone shows a more limited plasticization response and at a greater pressure of $\mathrm{CO}_{2}$, presumably reflecting the greater intrinsic rigidity of the pyrrolone compared to the imides and substituted polycarbonates.
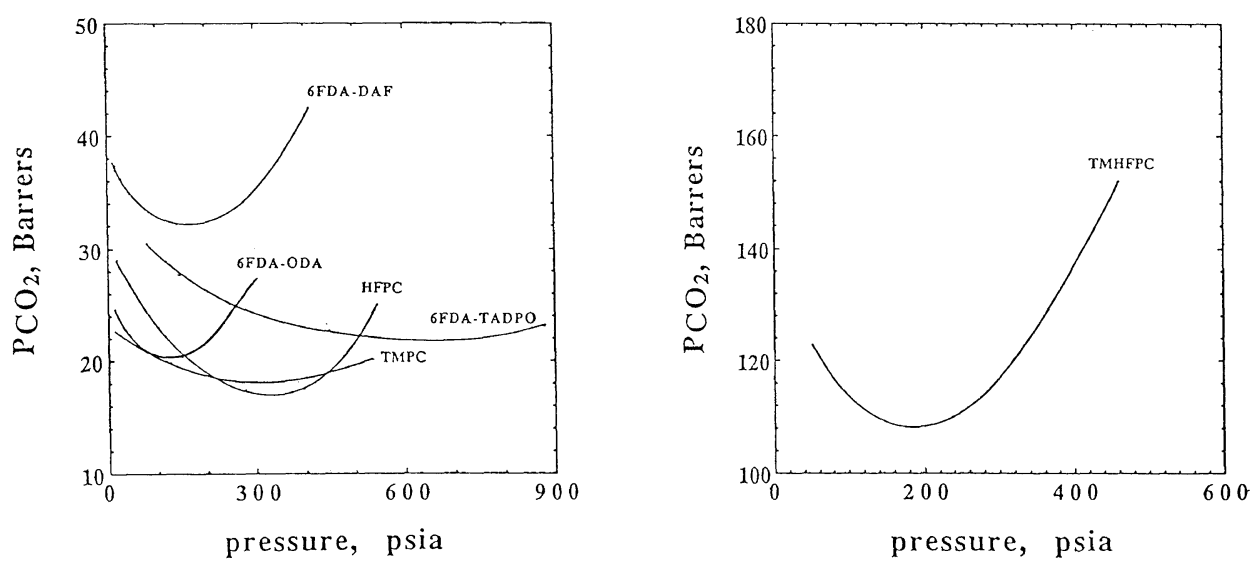

Figure 2: Pure gas $\mathrm{CO}_{2}$ permeability at $35^{\circ} \mathrm{C}$ versus pressure for materials in Table I.

In detailed analysis of the concentration dependence of the local effective diffusion coefficient of $\mathrm{CO}_{2}$ in the polypyrrolones, plasticization occurred at a sorption level of nearly $69[\mathrm{cc}(\mathrm{STP}) / \mathrm{cc}$ polymer], corresponding to $600 \mathrm{psia}$. For the polyimides plasticization occurred at only 50 [cc(STP)/cc polymer], corresponding to a 190 psia $\mathrm{CO}_{2}$ upstream pressure. An even more extreme situation has been shown to apply for the 
members of the polycarbonate family studied which plasticize at sorption levels near 30-35 [cc(STP)/cc polymer]. Because of the different sorption tendencies of the various materials, the various sorption levels correspond to rather different upstream pressures at the point of plasticization onset. In the case of the standard polycarbonate, this point of plasticization is prolonged until almost $600 \mathrm{psia}$, similar to the pressure of plasticization onset for the polypyrrolone. Under these conditions, however, the productivity of equivalent thicknesses of polycarbonate and polypyrrolone favor the polypyrrolone by almost a factor of four. The polypyrrolone is, therefore, intrinsically much more resistant to the effects of high $\mathrm{CO}_{2}$ sorption levels than the polyimides or the polycarbonates of similar productivity.

\section{Mixed Gas Permeation Results.}

The 50/50 feed mixture permeabilities of $\mathrm{CO}_{2}$ and the $\mathrm{CO}_{2} / \mathrm{CH}_{4}$ selectivities of the polypyrrolone, polyimides and the most rigid polycarbonate, TMHFPC, are shown in Fig. 3. The 6FDA-DAF polyimide has greater $\mathrm{CO}_{2}$ permeability than the polypyrrolone, but the polypyrrolone has a greater selectivity in the mixed gas than either of the polyimides. The selectivity decrease with respect to pressure increase is less for the polypyrrolone than for the polyimides. These results are contrary to the ideal permeability and selectivity results shown in Table V. Gas phase fugacity effects in the mixed gas can explain differences between pure gas and mixed gas results for a given polymer, but not the performance of one polymer relative to another in a mixed gas application. The most probable explanation for the reversal of the rank of the polymer permeabilities and selectivities is that the polypyrrolone is more resistant to plasticization than the polyimides, consistent with the discussion of local diffusion coefficients in the preceding section.

It seems reasonable that most of the deviations between pure and actual mixed gas feeds is attributable to enhanced mobility of the component one wishes to reject. Nevertheless, in the absence of actual mobility and solubility data for the mixtures, conclusions regarding the behavior of mixed feeds compared to the ratios of pure gases
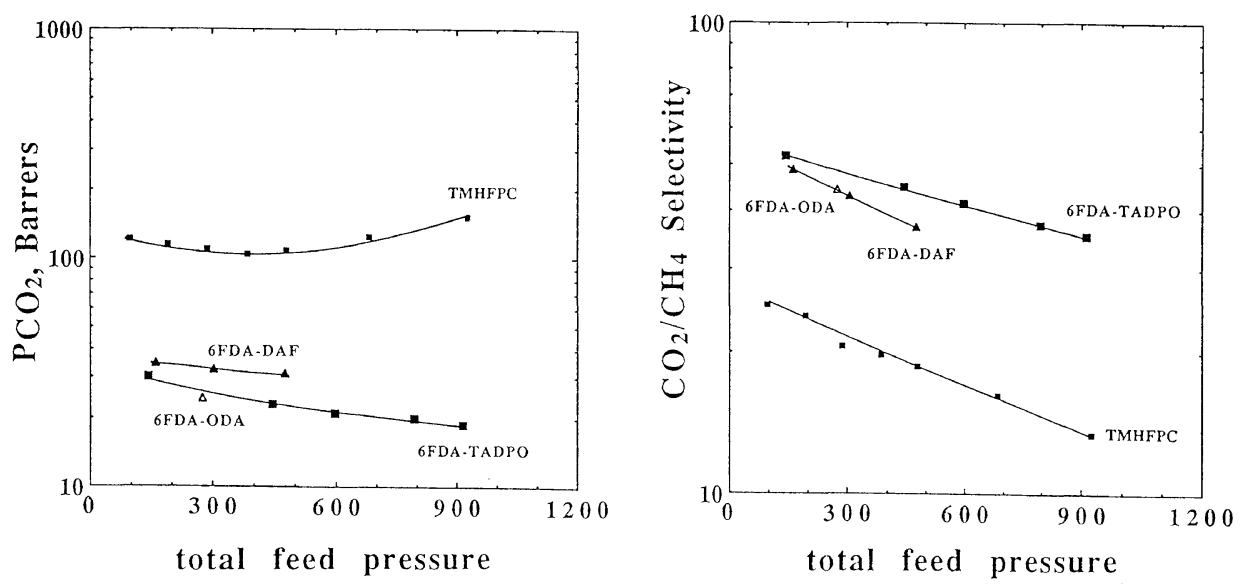

Figure 3: $\mathrm{CO}_{2}$ permeability and $\mathrm{CO}_{2} / \mathrm{CH}_{4}$ permslectivity at $35^{\circ} \mathrm{C}$ versus total pressure for a 50/50 mixture of the gases for various materials in Table I.

must be somewhat tentative at the present time. The mode of testing used in most laboratory characterizations of materials may provide a moderating factor that gives an overly optimistic view of the ability of the strongly plasticizing materials to maintain selectivity in mixed gas situations. Even when the upstream concentration exceeds that 
needed for local plasticization $(69,50$ and $35 \mathrm{cc}(\mathrm{STP}) / \mathrm{cc}$ polymer, for the pyrrolone, imide and carbonates, respectively), an unplasticized layer remains at the downstream face of the membrane under vacuum.

One may consider the implications of the earlier hypothetical suggestions regarding the effects of free volume distribution favoring the mobility selectivity for the rigid packing inhibited polyimide in relation to plasticization. If the overall free volume fraction is equivalent for members of the three families considered here, it is felt that the regions of well packed segments in the polyimides and polypyrrolone would tend to have relatively less local free volume than the corresponding polycarbonate. This would render the polycarbonates intrinsically more susceptible to plasticization by additional free volume contributed from an equivalent number of locally dissolved penetrants. Moreover, at an equivalent total sorption level, the fractional accommodation of penetrants in regions of imperfect packing as opposed to the selective well packed regions would tend to be higher in the rigid packing inhibited matrices. In addition to the intrinsically higher resistance to torsional motions, therefore, such a distribution of free volume may also promote maintenance of mobility selectivity in the rigid matrix materials.

\section{SUMMARY}

For highly supercritical penetrants such as oxygen and nitrogen tailoring the intersegmental packing and intrasegmental resistance to motion allows achieving very attractive productivities without sacrificing selectivity. Combination of a long, flat and rigid segment with a significant packing inhibiting segment may promote sorption of all penetrants without affecting the solubility selectivity much. The coexistence of regions of well packed environments having relatively low local free volume in communication with local environments of relatively unoccupied volume distributed as packing defects may provide an optimum molecular level "topography " for high selectivity and productivity. It may be that high solubility is promoted by the packing disrupted regions that communicate via the highly size and shape selective regions of well packed subsegments.

With regard to plasticization resistance, the combination of a long, flat and rigid segment with a significant packing inhibiting segment also seems desirable. Low free volume regions of well packed segments would tend to require local sorption of a larger number of penetrants to undermine the size and shape selecting properties of these regions than in a matrix of equivalent, but more uniformly distributed total free volume fraction.

\section{ACKNOWLEDGEMENTS}

The authors gratefully acknowledge support of this work by the Department of Energy's Office of Basic Energy Science under Grant No. DE-FG05-86ER13507.

\section{REFERENCES}

1. W. J. Koros, G. K. Fleming, S. M. Jordan, and T. H. Kim and H. H. Hoehn, Prog. Polym. Sci., 13, 339 (1988).

2. O'Brien, K.C., Koros, W.J., Barbari, T.A. and Sanders, E.S., J. Memb. Sci., 29, 229 (1986).

3. R. T. Chern, W. J. Koros, E. S. Sanders, S. H. Chen and H. B. Hopfenberg, in ACS Symposium Series 223 , "Industrial Gas Separations", ed. by

T. E. Whyte Jr., C. M. Yon, and E. H. Wagener, pg. 47 (1983).

4. T. H. Kim, W. J. Koros, G. R. Husk, and K. C. O'Brien, J. Membr. Sci.., 37, 45 (1988).

5. M. W. Hellums, W. J. Koros, G. R. Husk, and D. R. Paul, J. Membr. Sci.., 46, 93 (1989)

6. M. R. Coleman and W. J. Koros, J. Membr. Sci.., 50, 285 (1990). 
7. A. Bondi, "Physical Properties of Molecular Crystals, Liquids and Gases", Ch. 3 and 4, Wiley, New York (1968).

8. Van Krevelen, D. W. and Hoftyzer, P. J., "Properties of Polymers", 2nd Edition, Ch. 4, Elsevier, New York, (1976).

9. D. R. B. Walker and W. J. Koros, "Transport Characterization of a Polypyrrolone for Gas Separations", J. Membr. Sci.., in press. 